Special issue, XLVI Extraordinary Congress of Polish Physicists, Warsaw, Poland, October 16-18, 2020

\title{
Radiative Neutron Capture Cross-Section Measurement of Ge Isotopes at n_TOF CERN Facility and Its Importance for Stellar Nucleosynthesis
}

A. GaWlik ${ }^{a, *}$, C. Lederer-Woods ${ }^{b}$, J. Andrzejewski $^{a}$, J. Perkowski ${ }^{a}$,

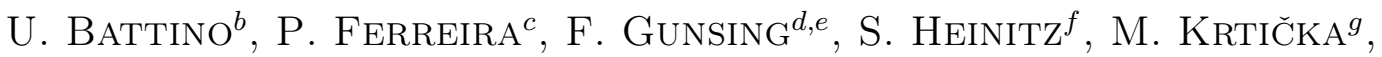

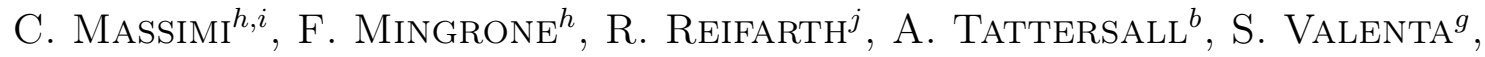

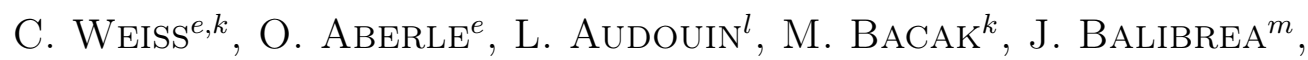
M. Barbagallo ${ }^{n}$, S. Barros ${ }^{c}$, V. BéCAres ${ }^{m}$, F. BeČvá $\check{R}^{g}$, C. Beinrucker $^{j}$, E. Berthoumieux ${ }^{d}$, J. Billowes ${ }^{o}$, D. Bosnar $^{p}$, M. BrugGer $^{e}$, M. CaAmaño ${ }^{q}$, F. Calviño ${ }^{r}$, M. Calviani ${ }^{e}$, D. Cano-Ott ${ }^{m}$, R. Cardella $^{e}$, A. Casanovas $^{r}$, D.M. Castelluccio ${ }^{s, h}$, F. Cerutti ${ }^{e}$, Y.H. Chen $^{l}$, E. Chiaveri ${ }^{e}$, N. Colonna ${ }^{n}$, G. Cortés ${ }^{r}$, M.A. Cortés-Giraldo ${ }^{t}$, L. Cosentino ${ }^{u}$, L.A. Damone ${ }^{n, v}$, M. Diakaki ${ }^{d}$, M. Dietz ${ }^{b}$, C. Domingo-Pardo ${ }^{w}$, R. Dressler ${ }^{f}$, E. Dupont $^{d}$,

I. Durán ${ }^{q}$, B. Fernández-Domínguez ${ }^{q}$, A. Ferrari $^{e}$, P. Finocchiaro $^{u}$,

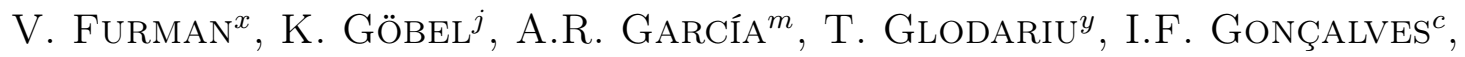
E. González-Romero ${ }^{m}$, A. Goverdovski ${ }^{z}$, E. Griesmayer $^{k}$, C. Guerrero $^{t}$, H. HARAdA ${ }^{a a}$, T. HeFtrich ${ }^{j}$, J. HeYse ${ }^{b b}$, D.G. JenKins ${ }^{c c}$, E. JerichA ${ }^{k}$,

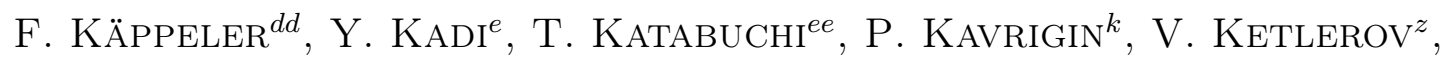

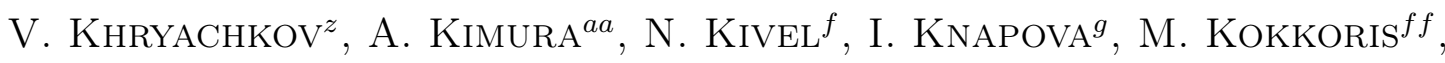
E. Leal-Cidoncha ${ }^{q}, \mathrm{H} . \mathrm{LeEB}^{k}$, J. Lerendegui-Marco ${ }^{t}, \mathrm{~S} . \mathrm{Lo} \mathrm{MeO}^{s, h}$, S.J. Lonsdale ${ }^{b}$, R. Losito $^{e}$, D. Macina ${ }^{e}$, T. Martínez $^{m}$, P. Mastind $^{g}$,

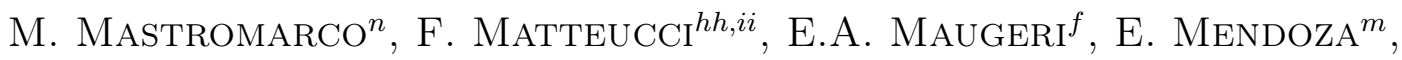

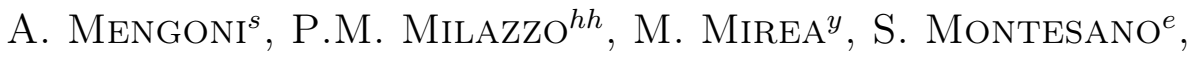

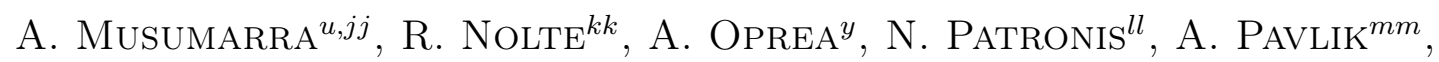
J.I. Porras ${ }^{e, n n}$, J. Praena ${ }^{t, n n}$, J.M. Quesada ${ }^{t}$, K. RAJeEV ${ }^{o o}$, T. RAuscheR ${ }^{p p, q q}$, A. Riego-Perez ${ }^{r}$, P.C. Rout ${ }^{o o}$, C. Rubbia ${ }^{e}$, J.A. Ryan ${ }^{o}$, M. Sabaté-Gilarte $^{e, t}$,

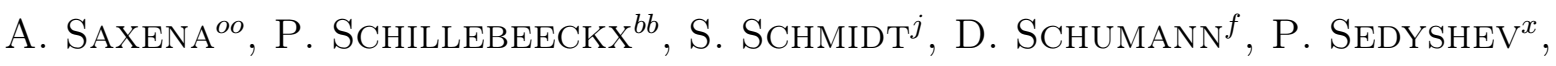

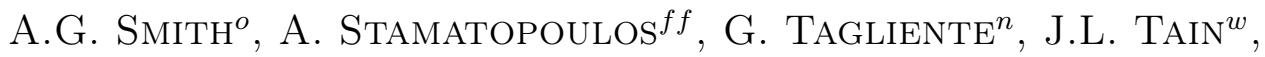

A. TARifeño-Saldivia ${ }^{w}$, L. TASsan-Got ${ }^{l}$, A. Tsinganis $^{f f}$, G. VAnnini $^{h, i}$,

V. VAriale ${ }^{n}, \mathrm{P} . \mathrm{VAZ}^{c}, \mathrm{~A} . \mathrm{Ventura}^{h}, \mathrm{~V} . \mathrm{Vlachoudis}^{e}, \mathrm{R}$ Vlastou $^{f f}$,

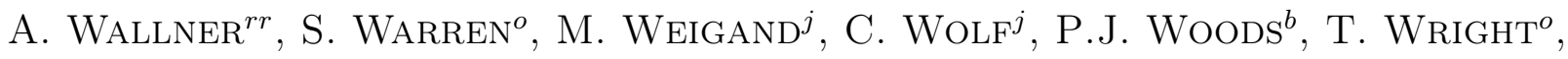

P. ŽugeC ${ }^{p, e}$ And The N_TOF COllaboration (WWW.CERn.CH/NTOF)

\footnotetext{
${ }^{a}$ University of Lodz, Poland

${ }^{b}$ School of Physics and Astronomy, University of Edinburgh, United Kingdom

${ }^{c}$ Instituto Superior Técnico, Lisbon, Portugal

${ }^{d}$ CEA Irfu, Université Paris-Saclay, F-91191 Gif-sur-Yvette, France

${ }^{e}$ European Organization for Nuclear Research (CERN), Switzerland

${ }^{f}$ Paul Scherrer Institut (PSI), Villingen, Switzerland

${ }^{g}$ Charles University, Prague, Czech Republic

${ }^{h}$ Istituto Nazionale di Fisica Nucleare, Sezione di Bologna, Italy
} 
${ }^{i}$ Dipartimento di Fisica e Astronomia, Università di Bologna, Italy

${ }^{j}$ Goethe University, Frankfurt, Germany

${ }^{k}$ Technische Universität Wien, Austria

'Institut de Physique Nucléaire, CNRS-IN2P3, Univ. Paris-Sud, Université Paris-Saclay, F-91406 Orsay Cedex, France

${ }^{m}$ Centro de Investigaciones Energéticas Medioambientales y Tecnológicas (CIEMAT), Spain

${ }^{n}$ Istituto Nazionale di Fisica Nucleare, Sezione di Bari, Italy

${ }^{\circ}$ University of Manchester, United Kingdom

${ }^{p}$ Department of Physics, Faculty of Science, University of Zagreb, Zagreb, Croatia

${ }^{q}$ University of Santiago de Compostela, Spain

${ }^{r}$ Universitat Politècnica de Catalunya, Spain

s Agenzia Nazionale per le Nuove Tecnologie (ENEA), Bologna, Italy

${ }^{t}$ Universidad de Sevilla, Spain

${ }^{u}$ INFN, Laboratori Nazionali del Sud, Catania, Italy

${ }^{v}$ Dipartimento di Fisica, Università degli Studi di Bari, Italy

${ }^{w}$ Instituto de Física Corpuscular, CSIC - Universidad de Valencia, Spain

${ }^{x}$ Joint Institute for Nuclear Research (JINR), Dubna, Russia

${ }^{y}$ Horia Hulubei National Institute of Physics and Nuclear Engineering, Romania

${ }^{z}$ Institute of Physics and Power Engineering (IPPE), Obninsk, Russia

aa Japan Atomic Energy Agency (JAEA), Tokai-mura, Japan

${ }^{b b}$ European Commission, Joint Research Centre, Geel, Retieseweg 111, B-2440 Geel, Belgium

${ }^{c c}$ University of York, United Kingdom

${ }^{d d}$ Karlsruhe Institute of Technology, Campus North, IKP, 76021 Karlsruhe, Germany

ee Tokyo Institute of Technology, Japan

ff National Technical University of Athens, Greece

${ }^{g g}$ Istituto Nazionale di Fisica Nucleare, Sezione di Legnaro, Italy

${ }^{h h}$ Istituto Nazionale di Fisica Nucleare, Sezione di Trieste, Italy

${ }^{i i}$ Dipartimento di Astronomia, Università di Trieste, Italy

${ }^{j j}$ Dipartimento di Fisica e Astronomia, Università di Catania, Italy

${ }^{k k}$ Physikalisch-Technische Bundesanstalt (PTB), Bundesallee 100,

38116 Braunschweig, Germany

${ }^{l l}$ University of Ioannina, Greece

${ }^{m m}$ University of Vienna, Faculty of Physics, Vienna, Austria

${ }^{n n}$ University of Granada, Spain

oo Bhabha Atomic Research Centre (BARC), India

${ }^{p p}$ Centre for Astrophysics Research, University of Hertfordshire, United Kingdom

${ }^{q q}$ Department of Physics, University of Basel, Switzerland

${ }^{r r}$ Australian National University, Canberra, Australia

Doi: 10.12693/APhysPolA.139.383

*e-mail: aleksandra.gawlik@uni.lodz.pl

This manuscript summarizes the results of radiative neutron capture cross-section measurements on two stable germanium isotopes, ${ }^{70} \mathrm{Ge}$ and ${ }^{73} \mathrm{Ge}$. Experiments were performed at the $\mathrm{n}$ TOF facility at CERN via the time-of-flight technique, over a wide neutron energy range, for all stable germanium isotopes $\left(70,72,73,74\right.$, and 76). Results for ${ }^{70} \mathrm{Ge}[$ Phys. Rev. C 100, $045804(2019)]$ and ${ }^{73} \mathrm{Ge}[$ Phys. Lett. B 790, 458 (2019)] are already published. In the field of nuclear structure, such measurements allow to study excited levels close to the neutron binding energy and to obtain information on nuclear properties. In stellar nucleosynthesis research, neutron induced reactions on germanium are of importance for nucleosynthesis in the weak component of the slow neutron capture processes.

topics: n_TOF, neutron time-of-flight, neutron capture cross-section, MACS

\section{Introduction}

Neutron capture reactions in stars are responsible for forming about $99 \%$ of the nuclei heavier than iron. The slow and rapid neutron capture processes contribute almost equally to the overall abundances. The $s$ process takes place when neutron densities are low $\left(10^{8} \mathrm{n} \mathrm{cm}^{-3}\right)$ and radioactive decay is faster than subsequent neutron capture on radionuclides [3]; when neutron densities are high $\left(>10^{20} \mathrm{n} \mathrm{cm}^{-3}\right)$ nuclei can capture many neutrons, creating nuclides far from the valley of stability, and then decay by $\beta^{-}$cascades (the so-called $r$ process [4]). The weak component of the $s$ process takes place in massive stars (>8 solar masses $M_{\odot}$ ) and forms isotopes of mass 


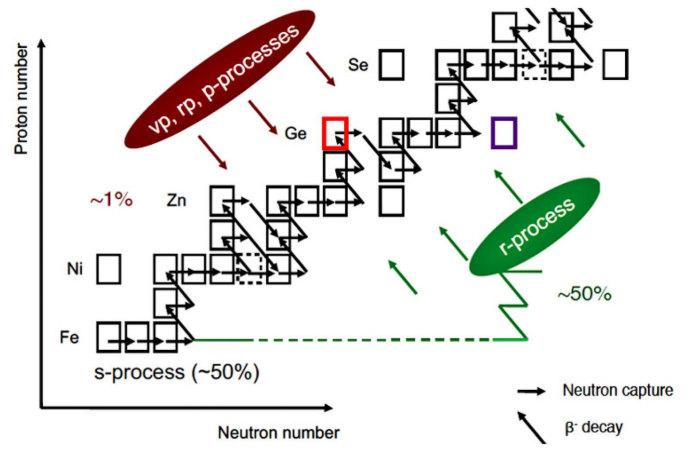

Fig. 1. Nucleosynthesis path of the $s$ process going along the stability valley. Solid boxes represent stable isotopes. Slow neutron captures and following $\beta$ decays are marked by arrow lines. Red box indicates the " $s$-only" ${ }^{70} \mathrm{Ge}$ isotope, purple box indicates the " $r$-only" ${ }^{76} \mathrm{Ge}$ isotope.

number between 56 and 90. In this scenario, neutrons are produced by ${ }^{22} \mathrm{Ne}(\alpha, \mathrm{n})^{25} \mathrm{Mg}$ reaction [5] in two different burning stages of stellar evolution. In massive stars, ${ }^{14} \mathrm{~N}$ is produced via the $\mathrm{CNO}$ cycle, and then converted to ${ }^{22} \mathrm{Ne}$ by the reaction sequence ${ }^{14} \mathrm{~N}(\alpha, \gamma){ }^{18} \mathrm{~F}\left(\beta^{+} \nu\right){ }^{18} \mathrm{O}(\alpha, \gamma){ }^{22} \mathrm{Ne}$. Towards the end of He core burning, at temperatures of about $0.3 \mathrm{GK}$, neutrons are released by the ${ }^{22} \mathrm{Ne}(\alpha, \mathrm{n})^{25} \mathrm{Mg}$ reaction. If some ${ }^{22} \mathrm{Ne}$ survives the helium burning stage, ${ }^{22} \mathrm{Ne}(\alpha, \mathrm{n}){ }^{25} \mathrm{Mg}$ source is reactivated at temperatures of about 1 GK during carbon shell burning [6-10].

Recent studies indicate that the weak $s$ process is responsible for about $80 \%$ of the total germanium abundance [11]. On the other hand, $12 \%$ of germanium is produced in the main $s$ process in asymptotic giant branch stars, with mass of about 1-5 $M_{\odot}$, during their thermal pulses. The remaining $8 \%$ is thought to be produced by primary explosive nucleosynthesis processes [11].

In stars, neutrons are thermalized very fast and their velocities can be described by the MaxwellBoltzmann distribution. Therefore, the key quantity to predict abundances produced in the $s$ process is the Maxwellian averaged cross-section (MACS). To study the $s$ process nucleosynthesis for the weak component, MACS values should be known up to $k_{\mathrm{B}} T$ values of about $100 \mathrm{keV}$.

Figure 1 shows the $s$ process path in the region of interest. The stable isotope with the lowest mass is ${ }^{70} \mathrm{Ge}$, which plays a special role as " $s$-only" nuclide (with minor contributions from proton-rich nucleosynthesis processes) since it is shielded from the $r$ process production by its stable isobar ${ }^{70} \mathrm{Zn} \mathrm{[1].}$ It suggests that ${ }^{70} \mathrm{Ge}$ is mainly produced in the $s$ process and its abundance can be used as a reference to compare to solar abundances.

On the other hand, ${ }^{76} \mathrm{Ge}$ due to its position on the path can be treated as an " $r$-only" isotope since it is shielded from the $s$ process by the unstable ${ }^{75} \mathrm{Ge}$ with short half-life of $83 \mathrm{~min}$. In addition, germanium is commonly used as detector material, and hence neutron induced reactions on germanium are important for low-background experiments using HPGe detectors [12]. The data for ${ }^{76} \mathrm{Ge}$ is currently under analysis.

\section{Experimental setup and measurement}

The measurements of the $(n, \gamma)$ cross-section of stable Ge isotopes at $n \_$TOF were performed over a wide neutron energy range from $\mathrm{eV}$ to several hundreds of $\mathrm{keV}$. The $\mathrm{n}$ - TOF facility is a part of the CERN accelerator complex, shown in Fig. 2. In the first accelerator, LINAC, protons are accelerated to energies of $1.4 \mathrm{GeV}$. Protons of energy up to $20 \mathrm{GeV}$ from Proton Synchrotron are sent to the $\mathrm{n}$ - TOF spallation target $\left(7 \times 10^{12}\right.$ protons per pulse with $0.8 \mathrm{~Hz}$ repetition rate). An intense neutron beam is produced by spallation reactions impinging on a cylindrical 1.3-tonne lead target $(40 \mathrm{~cm}$ length, $60 \mathrm{~cm}$ diameter) [13]. The moderated neutron spectrum ranges from thermal energies $(25 \mathrm{meV})$ to several $\mathrm{GeV}$.

Measurements were performed at Experimental Area 1 (EAR1), at a distance of $185 \mathrm{~m}$ from the spallation target, characterized by excellent energy resolution ranges from $3 \times 10^{-4}$ at $1 \mathrm{eV}$ to $3 \times 10^{-3}$ at $100 \mathrm{keV}$ [13]. The $\mathrm{n}_{-}$TOF facility is equipped with another experimental beam line, EAR2, with an approximately ten times shorter vertical flight path resulting in a higher flux but worse energy resolution.

For the detection of prompt $\gamma$ rays following capture reactions, we used $\mathrm{C}_{6} \mathrm{D}_{6}$ scintillation detectors, which have been specially optimised for extremely low sensitivity to scattered neutrons. Due to this feature, $\mathrm{C}_{6} \mathrm{D}_{6}$ detectors are most suitable for measuring isotopes with neutron capture cross-sections much lower than the elastic scattering cross-section. Samples used for the $(n, \gamma)$ measurements on Ge isotopes consisted of $\mathrm{GeO}_{2}$ powder pressed into pallets of cylindrical shape of $2 \mathrm{~cm}$ diameter. All samples were glued on to a sample holder with $6 \mu \mathrm{m}$ backing made of Mylar. Runs with an empty sample holder were performed to study the sample independent

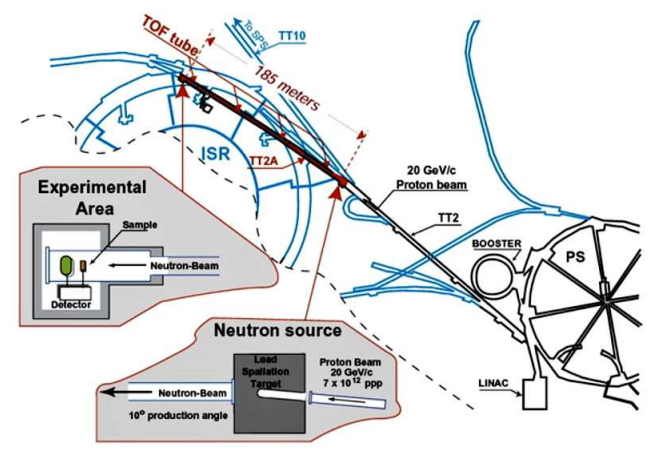

Fig. 2. Layout of the $\mathrm{n}_{-}$TOF facility at CERN. 
background. A metallic germanium sample of natural composition was measured to identify any contributions from other germanium isotopes present in the enriched samples.

\section{Data analysis}

As a first step, the dead-time corrected timeof-flight counting spectra were converted to neutron energy spectra with the known neutron timeof-flight and flight path length. The flight path length was determined by calibrating against well known low energy resonances in the ${ }^{197} \mathrm{Au}(\mathrm{n}, \gamma)$ reaction [14]. The limitations caused by the experimental setup itself (neutron energy resolution), allowed us to parameterize resonances up to $25 \mathrm{keV}$ and $14 \mathrm{keV}$ in the case of ${ }^{70} \mathrm{Ge}$ and ${ }^{73} \mathrm{Ge}$ isotope, respectively.

The experimental capture yield can be described as

$$
Y\left(E_{n}\right)=f_{N}\left(E_{n}\right) \frac{C\left(E_{n}\right)-B\left(E_{n}\right)}{\epsilon_{c} \Phi\left(E_{n}\right)},
$$

where $C\left(E_{n}\right)$ is the count spectrum of the measured sample, $B\left(E_{n}\right)$ is the background, $\epsilon_{c}$ is the detection efficiency, $\Phi$ is the neutron flux, and $f_{N}\left(E_{n}\right)$ is a normalisation factor.

Figure 3 shows the experimental capture yield compared to the yield predicted by ENDF/BVIII [15] (taking into account all the experimental effects). As can be seen, many new resonances are observed.

The background $B\left(E_{n}\right)$ at the n TOF facility consists of the following components: samplerelated background, beam-related background and ambient background unrelated to the beam. The environmental background is present during all measurements. It is due to natural radioactivity and cosmic rays and it is determined in runs without the beam. Beam-related background is mainly caused by interactions between the neutron beam and material in the experimental area and it is determined by measurements with an empty sample

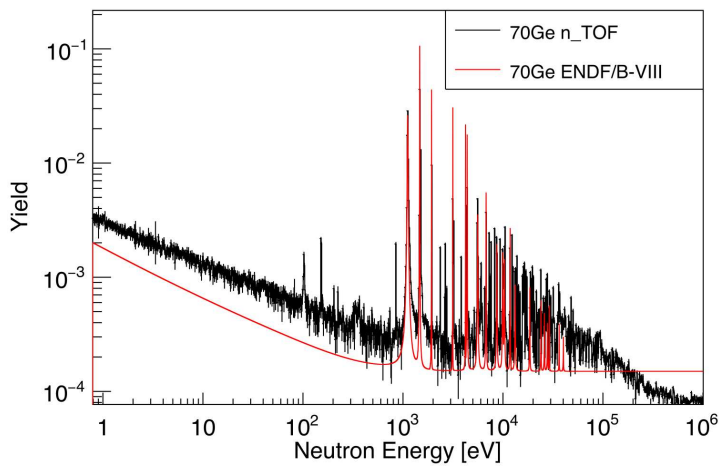

Fig. 3. Experimental capture yield of ${ }^{70} \mathrm{Ge}(\mathrm{n}, \gamma)$ reaction in comparison with ENDF/B-VIII capture yield.

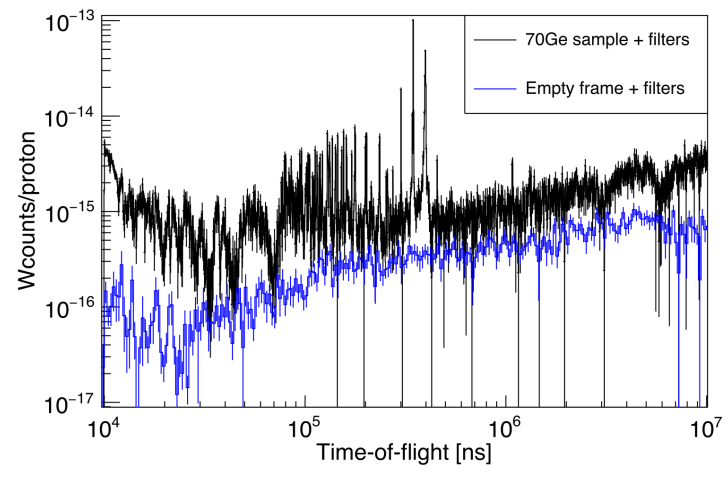

Fig. 4. ${ }^{70}$ Ge time-of-flight spectrum compared to empty sample holder, both measured with $\mathrm{Al}$ filter in the beam. The counting spectra are weighted and normalized to the total number of protons.

holder. Sample-related background is specifically caused by neutrons scattered off the sample. Usually, the capture probability is a few orders of magnitude lower than the scattering probability and thus many neutrons may be scattered from the sample. Scattered neutrons can be captured by the surrounding materials and cause additional background events. In order to correct for the background due to neutron scattering, runs with neutron filters in the beam were performed.

Figure 4 shows the ${ }^{70} \mathrm{Ge}$ spectrum as well as the empty sample holder spectrum, both measured with an Al filter in the beam. The filter was made of aluminum of proper thickness to capture all neutrons from the beam at certain resonance energies. Any events in the dips of these resonances are produced by background reactions. The aluminum filter has those dips at around 35, 86, and $150 \mathrm{keV}$ neutron energy. The background is scaled to these dips which are called black resonances.

The ${ }^{197} \mathrm{Au}$ sample was used to obtain a normalisation factor $f_{N}$ which corrects the capture yield since the diameter of the neutron beam is larger than the diameter of the capture sample. The sample used for this purpose had the same diameter as the Ge samples. The normalisation was determined using the ${ }^{197} \mathrm{Au}$ resonance at $4.9 \mathrm{eV}$ neutron energy, employing the saturated resonance method [16]. The detection efficiency was taken into account using the pulse height weighting technique (PHWT). It is based on applying pulse height dependent weights to the data, to achieve a capture efficiency proportional to the excitation energy of the compound nucleus. Weighting functions were obtained in detailed simulations of the experimental setup using GEANT4 [17, 18]. The neutron flux $\Phi$ is measured in a separate campaign, using a variety of different detectors and well known reference reactions such as ${ }^{10} \mathrm{~B}(\mathrm{n}, \alpha)$ and ${ }^{6} \mathrm{Li}(\mathrm{n}, \alpha)$ [19].

Neutron resonances from $(n, \gamma)$ reactions on germanium isotopes were fitted with the multilevel, multi-channel R-matrix code SAMMY [20]. 


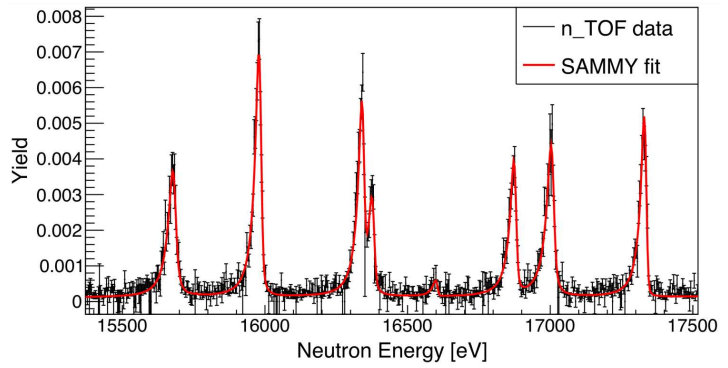

Fig. 5. Example for some SAMMY fits of the ${ }^{70} \mathrm{Ge}$ experimental capture yield. Solid line shows the result of SAMMY fit, dots are the experimental capture yield measured at $n_{-}$TOF.

SAMMY takes into account all experimental effects, such as broadening of resonance shape and self-shielding, and multiple scattering corrections.

In addition, sample impurities due to other Ge isotopes and oxygen were taken into account. From a single capture measurement only the capture kernel $k$ can be extracted. It is defined as:

$$
k=g \frac{\Gamma_{n} \Gamma_{\gamma}}{\Gamma_{n}+\Gamma_{\gamma}},
$$

where $g$ is a statistical factor for different spin combinations. It is given by

$$
g=\frac{(2 J+1)}{(2 s+1)(2 I+1)}
$$

where $J$ is the resonance spin, $s$ is the neutron spin and $I$ is the ground state spin of the target nucleus. The partial neutron and radiative widths, $\Gamma_{\mathrm{n}}$ and $\Gamma_{\gamma}$, respectively, and the energy were obtained with SAMMY. For known resonances, initial parameters were taken from the Atlas of Neutron Resonances [21]. Resonance structures can be observed until the experimental broadening is larger than the distance between subsequent resonances. This energy region is called the resolved resonance region (RRR). A few examples of ${ }^{70} \mathrm{Ge}$ resonance fits are shown in Fig. 5.

Average radiative width $\bar{\Gamma}_{\gamma}$ and the average resonance spacing $D_{0}$ were determined using the resonances in the RRR. Only strong resonances, for which the SAMMY fit yielded $\Gamma_{\mathrm{n}}>10 \Gamma_{\gamma}$, were chosen. Detailed results and averaged parameters for ${ }^{70} \mathrm{Ge}$ and ${ }^{73} \mathrm{Ge}$ can be found in [1] and [2], respectively. With increasing neutron energy, due to the worsening of the experimental resolution combined with lower statistics and increasing resonance widths, analysis of individual resonance parameters is no longer possible. This energy region is called the unresolved resonance region (URR). The level of the background also increases as a result of which a fraction of smaller resonances may be missed, resulting in an underestimation of the cross-section. The unresolved capture cross-section was obtained after subtraction of backgrounds due to neutron scattering and contributions due to impurities, and applying corrections for multiple scattering and selfshielding effect.

\section{Stellar capture cross-section}

We calculated Maxwellian averaged crosssections (MACS), defined as:

$$
\operatorname{MACS}=\frac{2}{\sqrt{\pi}} \frac{1}{\left(k_{\mathrm{B}} T\right)^{2}} \int_{0}^{\infty} \mathrm{d} E E \sigma(E) \mathrm{e}^{-E /\left(k_{\mathrm{B}} T\right)},
$$

using resonance information from SAMMY fits combined with the unresolved cross-section which we determined up to neutron energies of $300 \mathrm{keV}$. Final uncertainties in the MACS values are $\approx 5 \%$.

Figure 6 shows the ratio between experimental results obtained from $n_{-}$TOF data and values from the Karlsruhe Astrophysical Database of Nucleosynthesis in Stars [22], which is based on previous data and theoretical predictions.

In the case of ${ }^{70} \mathrm{Ge}$, there are only small changes in the resultant ${ }^{70} \mathrm{Ge}$ abundances produced in massive stars using the new ${ }^{70} \mathrm{Ge}$ MACS, about $3 \%$. The reason for that is a very good agreement with previous results published by Walter and Beer in 1985 [23]. In the case of ${ }^{73} \mathrm{Ge}$, MACSs are a factor about 1.5 larger than MACSs recommended to be used in stellar models, which were based on KADoNiS-1.0. It is worth mentioning that it was the first capture cross-section measurement on ${ }^{73} \mathrm{Ge}$ isotope. This results in a reduction of the ${ }^{73} \mathrm{Ge}$ abundances by over $30 \%$. The new data indicate that the germanium isotopic abundance pattern produced in the $s$ process in a low metallicity massive star is compatible with the isotopic abundance pattern of the solar system [2].

\section{Conclusions}

Neutron capture cross-sections on Ge have impact on abundances produced in the weak component of the slow neutron capture process. Recently, cross-sections on all stable Ge isotopes have been measured at the n_TOF facility at CERN. Results for ${ }^{70} \mathrm{Ge}$ and ${ }^{73} \mathrm{Ge}$ have already been published in $[1,2]$, while data analysis for the remaining isotopes is still ongoing.

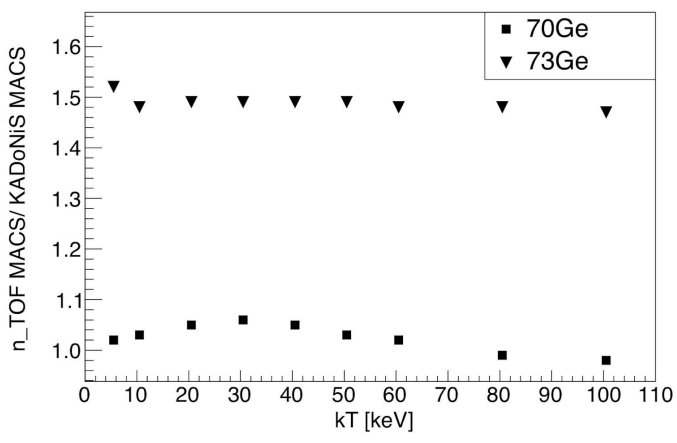

Fig. 6. Ratio between MACSs values obtained at $\mathrm{n}$-TOF $[1,2]$ and values from KADoNiS-1.0 database [22]. 


\section{Acknowledgments}

This work was supported by the Austrian Science Fund FWF (J3503), the Adolf Messer Foundation (Germany), the UK Science and Facilities Council (ST/M006085/1), and the European Research Council ERC-2015-StG No. 677497. We also acknowledge the support of the National Science Centre, Poland, under the grant UMO2016/22/M/ST2/00183, the MSMT of the Czech Republic and the Croatian Science Foundation under the project IP-2018-01-8570.

\section{References}

[1] A. Gawlik et al. (The n_TOF Collaboration), Phys. Rev. C 100, 045804 (2019).

[2] C. Lederer-Woods et al. (The n_TOF Collaboration), Phys. Lett. B 790, 458 (2019).

[3] R. Reifarth, C. Lederer, F. Käppeler, J. Phys. G 41, 053101 (2014).

[4] F.-K. Thielemann, A. Arcones, R. Käppeli et al., Prog. Part. Nucl. Phys. 66, 346 (2011).

[5] G. Cescutti, R. Hirschi, N. Nishimura, J.W. den Hartogh, T. Rauscher, A.St.J. Murphy, S. Cristallo, Mon. Not. R. Astron. Soc. 478, 4101 (2018).

[6] J.G. Peters, Astroph. J. 154, 225 (1968).

[7] R.G. Couch, A.B. Schmiedekamp, W.D. Arnett, Astroph. J. 190, 95 (1974).

[8] S.A. Lamb, W.M. Howard, J.W. Truran, I. Iben, Astroph. J. 217, 213 (1977).

[9] C.M. Raiteri, M. Busso, G. Picchio, R. Gallino, Astroph. J. 371, 665 (1991).

[10] C.M. Raiteri, M. Busso, G. Picchio, R. Gallino, L. Pulone, Astroph. J. 367, 228 (1991).

[11] M. Pignatari, R. Gallino, M. Heil, M. Wiescher, F. Käppeler, F. Herwig, S. Bisterzo, Astroph. J. 710, 1557 (2010).
[12] C. Lederer, J. Andrzejewski, M. Barbagallo et al. (The n_TOF Collaboration), Proposal to the ISOLDE and neutron timeof-flight committee, CERN, CERN-INTC2013-021 / INTC-P-381 (2013).

[13] C. Guerrero, A. Tsinganis, E. Berthoumieux et al. (The $\mathrm{n}_{-}$TOF collaboration), Eur. Phys. J. A 49, 27 (2013).

[14] C. Massimi, A. Borella, S. Kopecky, C. Lampoudis, P. Schillebeeckx, M.C. Moxon, G. Vannini, J. Kor. Phys. Soc. 59, 1689 (2011).

[15] D.A. Brown, M.B.Chadwick, R. Capote et al., Nucl. Data Sheets 148, 1 (2018).

[16] R.L. Macklin, J. Halperin, R.R. Winters, Nucl. Instr. Methods 164, 213 (1979).

[17] R.L. Macklin, R.H. Gibbons, Phys. Rev. 159, 1007 (1967).

[18] P. Schillebeeckx, B. Becker, Y. Danon et al., Nucl. Data Sheets 113, 3054 (2012).

[19] M. Barbagallo, C. Guerrero, A. Tsinganis et al. (The n_TOF Collaboration), Eur. Phys. J. A 49, 156 (2013).

[20] N.M. Larson, Technical report ORNL/ TM-9179/R8, Updated Users Guide for SAMMY: Multilevel R-Matrix Fits to Neutron Data Using Bayes' Equations, OakRidge National Laboratory, Oak Ridge (TN) 2008.

[21] S.F. Mughabghab, Atlas of Neutron Resonances, Elsevier, Amsterdam 2006, eBook.

[22] The Karlsruhe Astrophysical Database of Nucleosynthesis in Stars 1.0 (test version), latest release Kadonis-0.3; I. Dillmann, M. Heil, F. Käppeler, R. Plag, T. Rauscher, F.K. Thielemann, AIP Conf. Ser. 819, 123 (2006).

[23] G. Walter, H. Beer, Astron. Astrophys. 142, 268 (1985). 